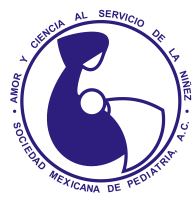

\title{
Duplicación de uretra: reporte de dos casos
}

\author{
Duplication of the urethra: report of two cases
}

\author{
Jenny Elizabeth Arboleda-Bustán,* Oriol Martín-Solé,* \\ Sonia Pérez-Bertólez,* Luis García-Aparicio* \\ * Sección de Urología Pediátrica. Servicio de Cirugía Pediátrica. Hospital Sant Joan de Déu. Universidad de Barcelona, Barcelona, España.
}

\begin{abstract}
RESUMEN
Objetivo: Presentar dos casos con duplicación uretral, los cuales se resolvieron mediante cirugía. Descripción de los casos: Fueron dos varones de tres y cuatro años de edad, con duplicación uretral tipo IA de acuerdo con clasificación de Effmann. Ambos se trataron con cirugía mediante exéresis de la uretra accesoria. Conclusión: La duplicación uretral es una malformación rara, más frecuente en varones. El manejo quirúrgico se reserva para casos sintomáticos.
\end{abstract}

Palabras clave: Duplicación de uretra, cirugía de uretra, clasificación de Effmann.

\section{INTRODUCCIÓN}

La duplicación uretral es una malformación poco frecuente, para el año 2012 se menciona que se habían publicado alrededor de 200 casos. ${ }^{1}$ Es más frecuente en el varón, pero cuando se presenta en la mujer se asocia a una duplicación de vejiga. ${ }^{2} \mathrm{La}$ diversidad de la clínica dificulta el diagnóstico, ya que los pacientes pueden ser asintomáticos o presentar uno o más de los siguientes síntomas: secreción mucosa, incontinencia, infección urinaria, retención urinaria recurrente, doble chorro miccional o cálculos. El diagnóstico diferencial se realiza con hipospadias, epispadias, obstrucción uretral. Para la confirmación del diagnóstico se puede requerir un cistograma miccional. ${ }^{3}$

\begin{abstract}
Objective: To present two cases with urethral duplication that were resolved by surgery. Description of the cases: We present two patients aged three and four years, with type IA urethral duplication according to the Effmann classification. Both were treated surgically by exeresis of the accessory urethra. Conclusion: Urethral duplication is a rare malformation, which occurs more frequently in men. Surgical management is reserved for symptomatic cases.
\end{abstract}

Keywords: Urethra duplication, urethral surgery, Effmann classification.

Existen diferentes formas, por lo que para distinguirlos se ha usado la clasificación descrita por Effmann que consta de tres tipos: ${ }^{4}$

Tipo I: duplicación incompleta en saco ciego, pero con dos formas, la IA corresponde a duplicación incompleta distal, la cual está abierta en el pene pero que no se comunica con la uretra. La IB es proximal, la cual está abierta desde el canal uretral y termina ciegamente en el tejido periuretral.

Tipo II: es la duplicación uretral permeable completa, se subdivide en IIA1, en la cual ambas uretras emergen de manera independiente la vejiga; mientras que IIA2 es duplicación incompleta, que se origina de una uretra proximal común, terminando en el perineo (duplicación en forma de Y). El tipo IIB es raro, en el

Correspondencia: Jenny Elizabeth Arboleda-Bustán, E-mail: elizabethab2011@gmail.com

Citar como: Arboleda-Bustán JE, Martín-Solé O, Pérez-Bertólez S, García-Aparicio L. Duplicación de uretra: reporte de dos casos. Rev Mex Pediatr. 2021; 88(4): 156-158. https://dx.doi.org/10.35366/102781 

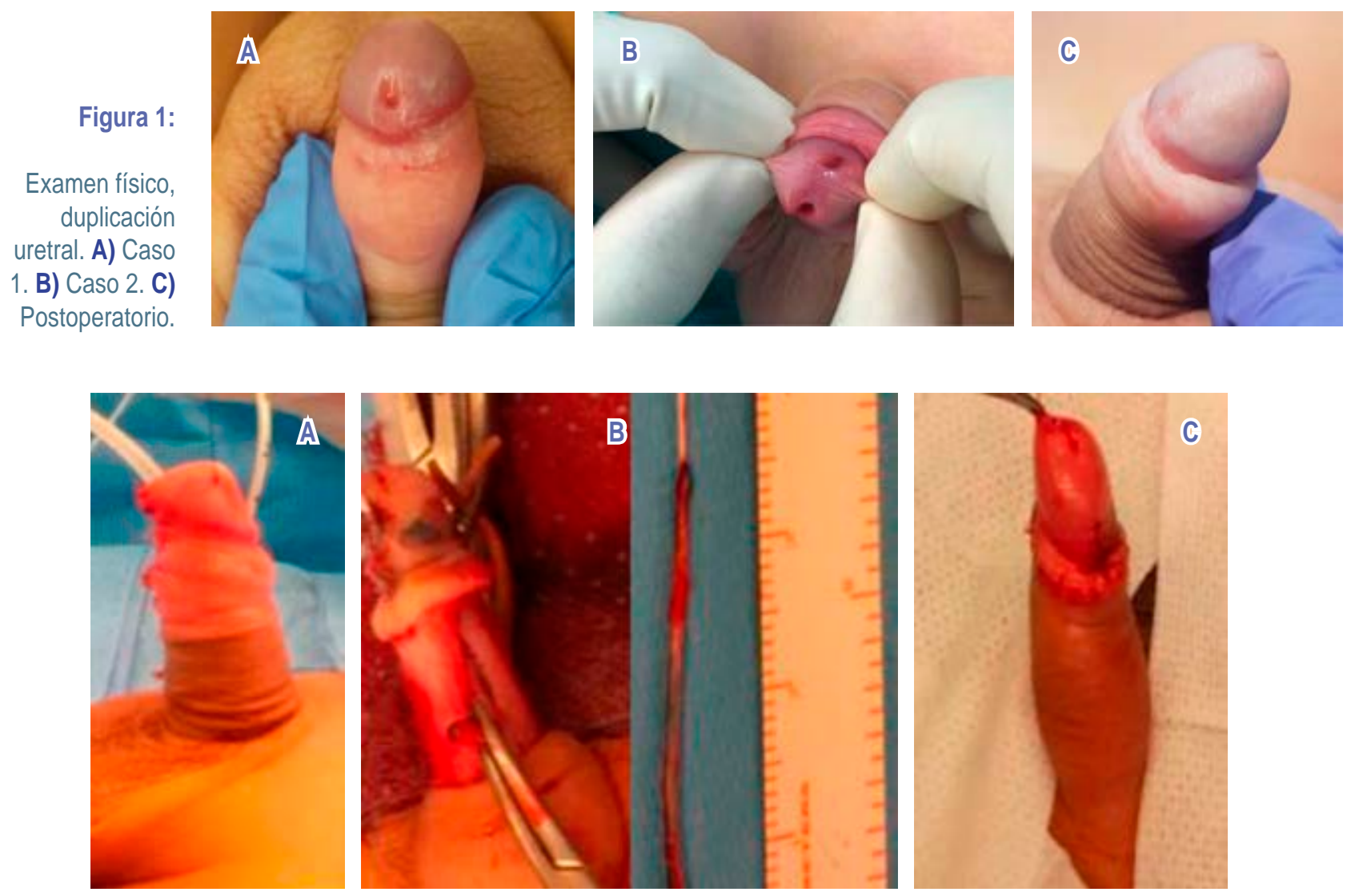

Figura 2: Intervención quirúrgica: A) Cateterización de ambas uretras. B) Disección y exéresis de la duplicación uretral, pieza quirúrgica. C) Aspecto final postquirúrgico.

que las dos uretras emergen de la vejiga o de la uretra posterior, uniéndose a un canal común distalmente.

Tipo III: es la duplicación de uretra con dos vejigas.

No parece existir un consenso sobre el origen, pero se ha propuesto que la causa embriológica de la duplicación uretral es por falla de la penetración del mesodermo lateral sobre las capas del ectodermo y endodermo de la membrana cloacal en la línea media. ${ }^{3,5,6}$

En cuanto al tratamiento, la cirugía se reserva para los pacientes sintomáticos. En este trabajo se presentan dos casos que requirieron tratamiento quirúrgico.

\section{PRESENTACIÓN DE LOS CASOS}

Caso 1: varón de cuatro años de edad que acude por doble meato uretral en la cara dorsal del glande (Figura 1A). Después de la realización de cistouretrografía miccional y uretrografía retrógrada a través de la duplicación uretral, se clasificó como tipo IA. Dadas las características se programó para realizar cirugía (la técnica se describe más adelante).

Caso 2: varón de tres años de edad que acude por doble meato uretral en la cara dorsal del glande. Una semana previa presentó balanitis (Figura 1B). Se realizó cistouretrografía miccional y uretrografía retrógrada a través de la duplicación uretral, clasificándola como tipo IA. Se realiza el mismo procedimiento quirúrgico descrito del caso previo. En la Figura 1C se observa el control postoperatorio; a dos años de seguimiento, no ha presentado complicaciones.

\section{TÉCNICA QUIRÚRGICA}

Se procede a realizar exéresis de la duplicación uretral: se canaliza la uretra verdadera con una sonda $8 \mathrm{Fr}$ y la uretra duplicada (falsa) con un catéter ureteral $3 \mathrm{Fr}$ (Figura 2A). Se realiza un degloving (desplegamiento) completo del pene, respetando la banda vasculonervio- 
sa del pene, así como disección de la fascia de Buck. Se identifica el fondo de saco de la duplicación instilando azul de metileno; por último, se realiza exéresis completa de la duplicación $(3.5 \mathrm{~cm})$ que termina en saco ciego (Figura 2B). Por último, se realiza plastia de piel (Figura 2C).

\section{DISCUSIÓN}

La duplicación de uretra se podría presentar tanto en el plano sagital como en el horizontal, aunque en el sagital es más común, como nuestros casos. 1,7-9

De acuerdo con la clasificación de Effmann, los dos casos que presentamos correspondieron al subtipo IA, en los cuales la uretra termina en saco ciego. Ésta puede presentarse en el plano sagital u horizontal, su incidencia es de $25 \%$. Los pacientes pueden ser asintomáticos o presentar secreción mucosa o purulenta. La más común es el tipo II con $62.5 \%$ de los casos. ${ }^{6,9,10}$ Mientras que el tipo III se presenta en $12.5 \%$, pero se puede asociar con malformación anorrectal, meningocele, complejo de extrofia-epispadias. ${ }^{11}$

En la duplicación uretral tipo I, la falla embriológica detectada es probablemente secundaria a la falla del desarrollo del tabique urorrectal. ${ }^{10,12-14}$

Algunos autores consideran que los casos con el tipo I (A o B) cuando son asintomáticos no tienen indicación quirúrgica. ${ }^{14,15}$ Pero si presenta secreción se tendrá que realizar la exéresis de la uretra accesoria. ${ }^{10,16}$ Otra opción es una neouretroplastia descrita por Podesta. ${ }^{17-19}$ Nosotros escogimos la exéresis completa de la uretra accesoria, que tiene la ventaja de prevenir infecciones por la acumulación de material, aunque otra razón es por estética. ${ }^{9}$

\section{AGRADECIMIENTOS}

Expresamos nuestro agradecimiento al Hospital Sant Joan de Déu por las facilidades prestadas para el presente trabajo.

\section{REFERENCIAS}

1. Montoya-Chinchilla R, Hidalgo-Agulló G, Romero-Hoyuela A, Rosino-Sánchez A. Duplicidad uretral. La importancia de una anamnesis y una exploración física detalladas. Rev Int Androl. 2013; 11:66-9.
2. Coleman RA, Winkle DC, Borzi PA. Urethral duplication: cases of ventral and dorsal complete duplication and review of the literature. J Pediatr Urol. 2010; 6(2): 188-191.

3. Ramírez-Ortega MÁ, Gamez-Torres O. Duplicación uretral con cálculo gigante. Informe de caso. Rev Med Inst Mex Seguro Soc. 2008; 46(2): 179-183.

4. Effmann EL, Lebowitz RL, Colodny AH. Duplication of the urethra. Radiology. 1976; 119(1): 179-185.

5. Pastor Navarro H, Carrion Lopez P, Martinez Ruiz J, Pastor Guzman JM, Salinas Sanchez AS, Virseda Rodriguez JA. Collateral urethral duplication in an adult. Arch Esp Urol. 2014; 67(4): 345-349.

6. Kang SK, Kim J, Lee YS, Han SW, Kim SW. Urethral duplication in male children: a study of 12 cases. J Pediatr Surg. 2020; 55(10): 2216-2220. doi: 10.1016/j.jpedsurg.2019.12.012.

7. Salle JLP, Sibai H, Rosenstein D, Brzezinski AE, Corcos J. Urethral duplication in the male: review of 16 cases. J Urol. 2000; 163(6): 1936-1940.

8. Urakami S, Igawa M, Shiina H, Shigeno K, Yoneda T, Yagi H. Congenital collateral urethral duplication in the frontal plane. $J$ Urol. 1999; 162(6): 2097-2098.

9. Middleton AW, Melzer RB. Duplicated urethra: an anomaly best repaired. Urology. 1992; 39(6): 538-540, 541-542.

10. Barrett DM, Malek RS, Kelalis PP. Problems and solutions in surgical treatment of 100 consecutive ureteral duplications in children. J Urol. 1975; 114(1): 126-130.

11. Perren F, Frey P. The exstrophy-epispadias complex in the duplicated lower urinary tract. J Urol. 1998; 159(5): 1681-1683.

12. Mane SB, Obaidah A, Dhende NP, Arlikar J, Acharya H, Thakur A et al. Urethral duplication in children: our experience of eight cases. J Pediatr Urol. 2009; 5(5): 363-367. doi: 10.1016/j. jpurol.2009.01.006.

13. Naparstek S, Abrams HJ, Sutton AP, Buchbinder MI. Complete duplication of male urethra in children. Urology. 1980; 16(4): 391392. doi: 10.1016/0090-4295(80)90146-6.

14. Lopes RI, Giron AM, Mello MF, Machado C, Neto B, Santos J et al. Urethral duplication type influences on the complications rate and number of surgical procedures. Int Braz J Urol. 2017; 43(6): 11441151.

15. Raffoul L, Rod J, Ravasse P, Blanc T, Lortat-Jacob S. Q-island flap urethroplasty: 1-stage procedure for reconstruction of Y-type urethral duplications in children. J Urol. 2015; 193(6): 2068-2072.

16. Sencan A, Yildiz M, Ergin M, Hoşgör M. A new variation of urethral duplication. Urology. 2013; 82(2): 451-453.

17. Podesta ML, Medel R, Castera R, Ruarte AC. Urethral duplication in children: Surgical treatment and results. J Urol. 1998; 160(5): 1830-1833.

18. Lima M, Destro F, Di Salvo N, Gargano T, Ruggeri G. Fate of males with urethral "Y-duplication": 40-year long follow-up in 8 patients. J Pediatr Surg. 2017; 52(8): 1335-1339.

19. Mohamed F, Jehangir S. Coexistent duplication of urethra and a refluxing ectopic ureter presenting as recurrent epididymo-orchitis in a child. BMJ Case Rep. 2017; 2017:bcr2017220278. doi: 10.1136/bcr-2017-220278.

Conflicto de intereses: los autores declaran que no tienen. 